\title{
Vielfalt der Frühlingsgeophyten in Kasachstan
}

\author{
Thomas STER
}

\begin{abstract}
Kazakhstan is a very interesting region for botanical excursions in march and april because of the many spring geophytes. The south east presents nearly all climatic and vegetation zones of the country from mountain vegetation to steppe vegetation and stone or sand deserts.
\end{abstract}

\section{Zusammenfassung}

Kasachstan ist vor allem wegen der vielen Frühlingsgeophyten ein botanisch interessantes Reiseziel in den Frühjahrsmonaten März und April. Der Südosten, ausgehend von Almaty, bietet nahezu alle Klima- und Vegetationszonen des Landes von den Vorbergen des Alatau über Steppen und ausgedehnte Fels- und Sandwüsten.
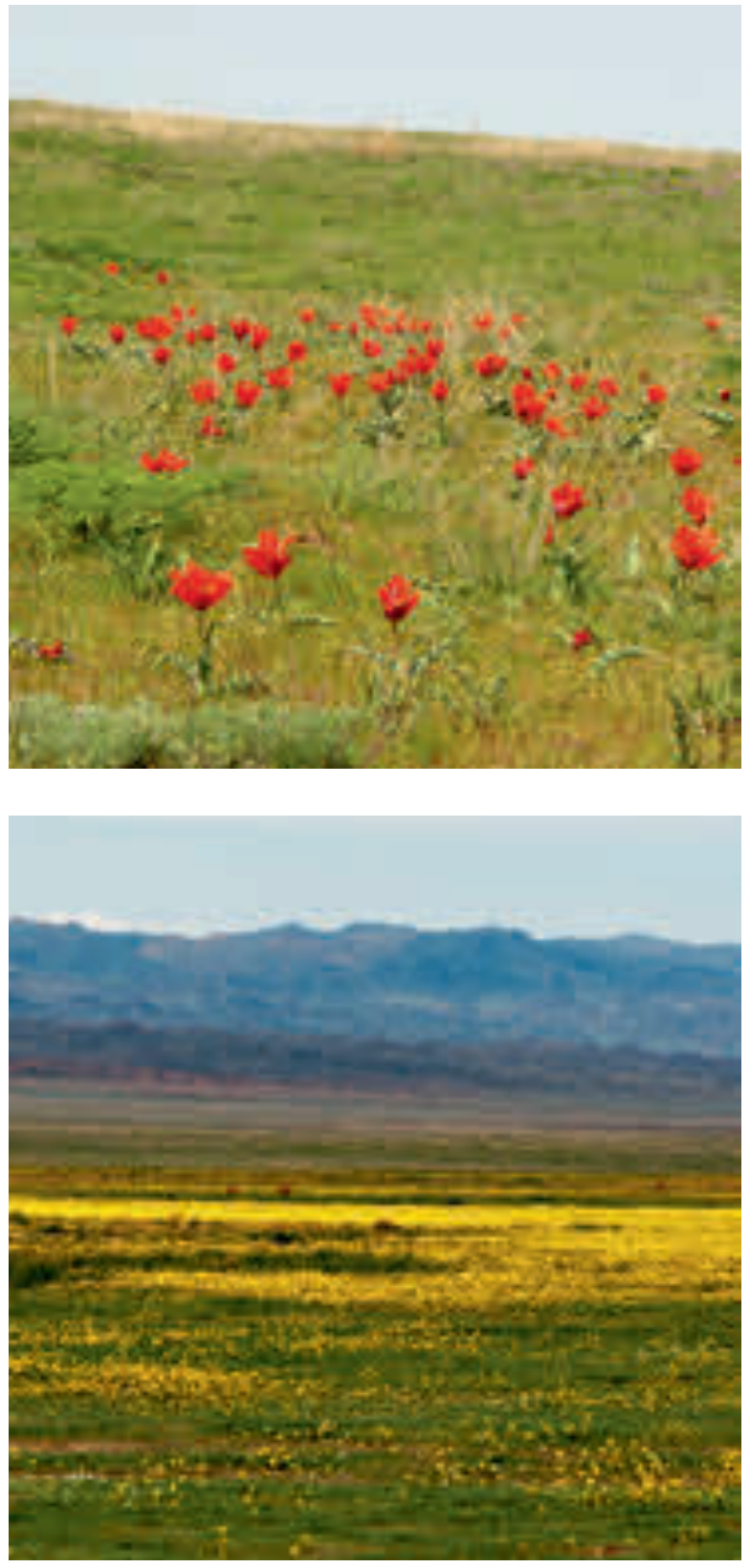

\section{Auf Exkursion in Kasachstan}

Der nachfolgende Beitrag basiert auf einer organisierten Exkursion der Österreichischen Gartenbaugesellschaft, an der auch der Autor teilgenommen hat. Insgesamt waren sieben Mitglieder der Gartenbaugesellschaft aus Österreich dabei, zudem drei deutsche Ornithologen. Die Exkursion fand vom 13. April bis 29. April 2013 statt, wobei besonders großes Interesse den Frühlingsgeophyten (Schwerpunkt Tulpen) in Kasachstan galt. Die örtliche Organisation und Begleitung erfolgte von Dagmar Schreiber (Almaty) und Stefan Flechtner (Bishkek). Botanisch betreut wurde die Gruppe über die Akademie der Wissenschaften in Bishkek/Kirgistan durch Georgij Laskov und Frau Almagül Kenderboeva. Ein ausführlicher Exkursionsbericht liegt beim Autor vor.

\section{Kasachstan, Verbreitungsschwerpunkt von Tulpen}

Im zeitigen Frühjahr, wenn die ersten botanischen Tulpen in unseren Gärten zu blühen beginnen, denkt kaum jemand daran, wo diese Frühlingsboten ihre Heimat haben. Die am häufigsten angebotenen Greigii-Tulpen (Tulipa greigii) und Seerosen-Tulpen (Tulipa kaufman-

Abb. 1 (oben): Tulipa greigii.

Abb. 2 (unten): Sogety-Hochebene mit Syrenia.

Abb. 3 (Seite 133): Kysylköl - Salzsee. 
niana) stammen wie die weitaus größte Zahl der Tulpenarten aus den Steppen- und Bergregionen Zentralasiens. Nirgendwo in Zentralasien findet man aber so viele Arten in ganz unterschiedlichen Lebensräumen wie in Kasachstan. Kasachstan ist das neuntgrößte Land der Erde und reicht vom Kaspischen Meer bis an die westlichen Grenzen Chinas. Es wäre aber zu kurz gegriffen, Kasachstan auf Tulpen zu reduzieren. Geschätzte 5700 Pflanzenarten kommen in Kasachstan vor, davon sind 700 endemisch. Die Flora dieses riesigen Landes im Herzen Asiens hält also viele Schätze bereit, die vor allem in den niederschlagsreichen Frühjahrsmonaten April und Mai die Steppen und Halbwüsten in ein Blütenmeer verwandeln, während gleichzeitig in den Bergregionen der Frühling erst erwacht.

\section{Landschaftliche Gliederung des Landes}

Klimatisch und landschaftlich lässt sich $\mathrm{Ka}$ sachstan ganz grob in vier Zonen unterteilen: 1. die Kaspische Senke mit teils feuchter Witterung; 2. die Gebirgsregion im Südosten mit dem Alatau, der Teil des Tian-Shan-Gebirges ist; 3. die Steppen- und Halbwüstengebiete mit einem typisch kontinentalen Klima und Randzonen, wo bedingt durch die Ausläufer des Alatau auch in den eher trockenen Jahreszeiten durch Nebel und Wolkenstau etwas mehr Niederschlag gegenüber den angrenzenden Steppengebieten fällt; 4 . der größte Teil des Landes mit kontinentalen Steppen und Halbwüsten.

Reisen nach Kasachstan beginnen meist mit dem Anflug auf die alte Hauptstadt Almaty (früher Alma Ata), die noch immer wirtschaftliches und kulturelles Zentrum ist. Seit 1997 ist die im Herzen Kasachstans liegende Stadt Astana Hauptstadt. Über den Süden der Stadt Almaty erhebt sich der im Frühling noch tief verschneite Transili Alatau, während im Norden die weiten Steppengebiete des Siebenstromlandes bis in die Vororte der Stadt reichen. Dementsprechend wirkt die Stadt nahezu zweigeteilt. Auf den Berghängen des Transili Alatau, wo einst große Apfelkulturen standen, die der Stadt den Namen gaben (Alma Ata wäre mit „Vater der Äpfel“ zu übersetzen), drängen sich jetzt noble Villen und besetzen fast unbemerkt die ungeschützten Randgebiete des $\mathrm{Na}$ tionalparks Ile-Alatau. Im Norden frisst sich die Stadt in das Steppengebiet.

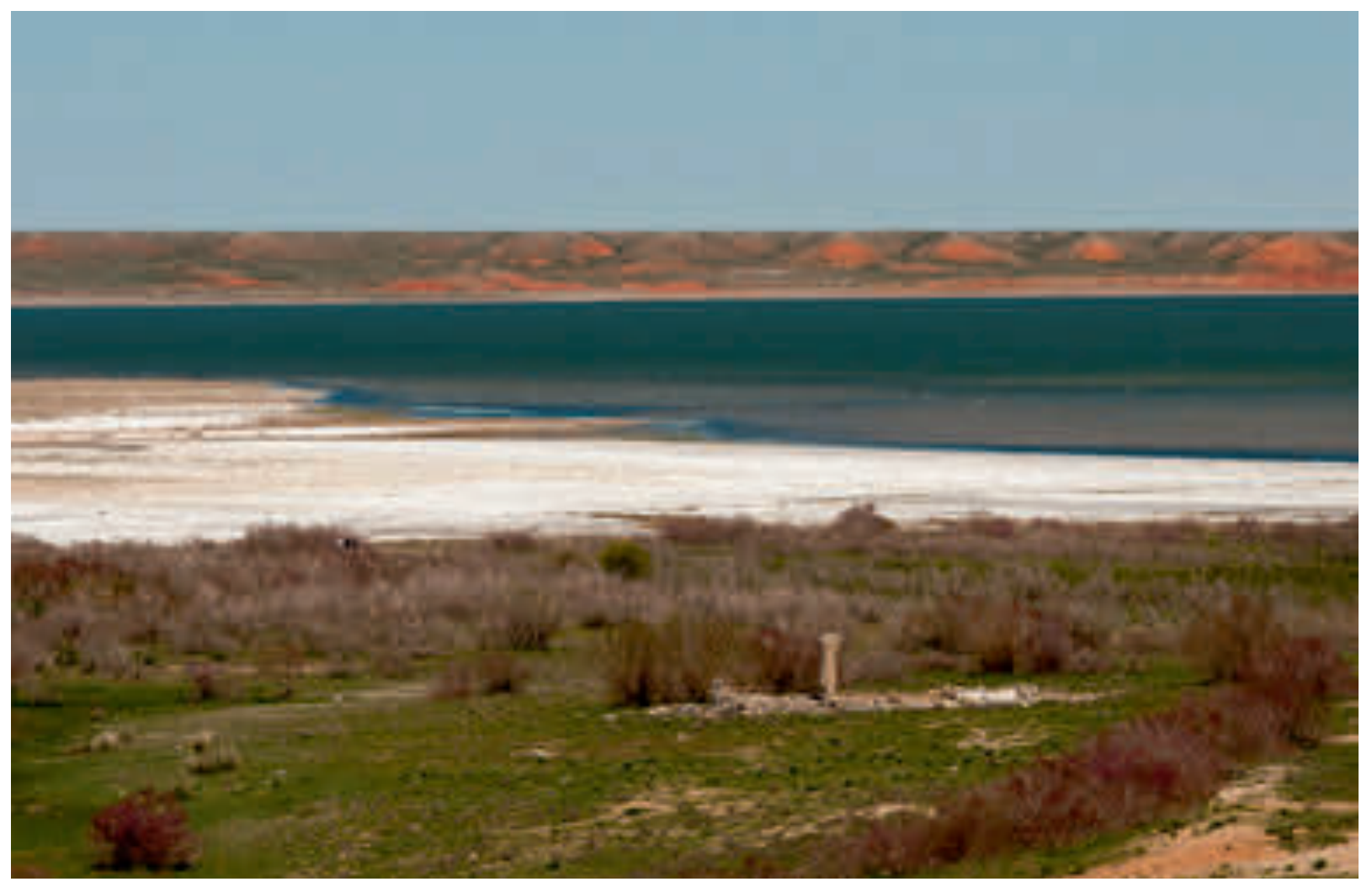




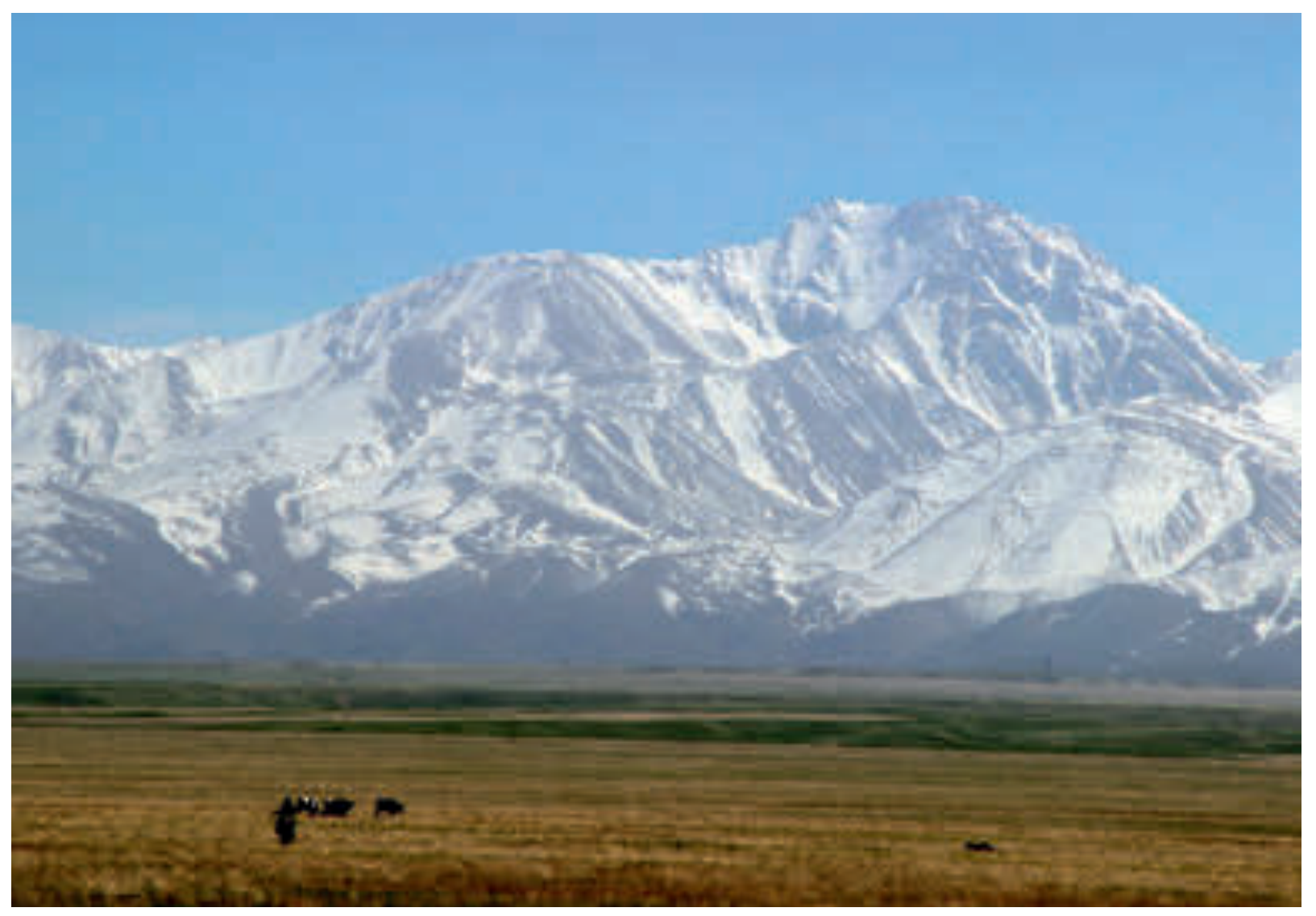

\section{Das Siebenstromland, die Wüsten Taukum und Mojynkum}

Nach der von Menschenhand hervorgerufenen ökologischen Katastrophe um den Aralsee ist der Balchaschsee zum größten Binnensee Kasachstans geworden. Er besteht aus dem nordöstlichen salzhaltigen Teil und dem südwestlichen Süßwassersee, die nur über eine schmale Verbindung (Uzun-Aral-Straße) miteinander in Kontakt stehen. Der geringe Salzgehalt des einen Teils des Sees wird durch die großen $\mathrm{Zu}$ flüsse sowie die geringe Durchmischung der beiden Teilseen verursacht, wodurch der Balchaschsee weltweit eine Besonderheit darstellt. Der wasserreiche, $1000 \mathrm{~km}$ lange, im TianShan-Gebirge entspringende Ili-Fluss speist mit einem breiten Delta den südwestlichen Teil. Nördlich von Almaty bei Kapaschagai wird der Ili zur Energiegewinnung aufgestaut. In der umgebenden Felssteppe blühen im April unzählige meist gelbblühende Tulipa kolpakovskiana. Große rote, selten gelbe Blüten bildet die ebenso hier auftretende Tulipa albertii. Außerdem gedeihen hier Ixilirion tataricum und die auffälligen, bis einen Meter hohen Sträucher von Spiraea hypericifolia.

Auf den ebenen windgefegten Flächen, wo schon im April die meisten Geophyten verblüht sind, blühten während der Exkursionszeit noch einige Iris tenuifolia und Rindera tetraspis. Der Übergang in die Sandwüste wird manchmal durch mächtige Dünen gebildet. Teilweise vom Treibsand zugeweht, blüht eine zarte weißblühende Tulpe (Tulipa buhseana) zwischen scheinbar dürren Saxaulsträuchern (Haloxylon ammodendron). Es überraschen die Anpassungsmöglichkeiten der Tulpen, die auch in den Sandwüstenböden mit einigen Arten vertreten sind. Speziell angepasst sind z. B. Tulipa behmanniana und T. buhseana. So befindet sich die Zwiebel der letztgenannten üblicherweise im Sandboden. Die Zwiebel sowie der etwa $30 \mathrm{~cm}$ lange Schaft sind durch eine dunkelbraune, wollige Tunika vor den Witterungseinflüssen geschützt.

Die Ausläufer des Alatau bilden breite, über eintausend Meter hohe Hügelketten wie die Chu-Ili-Berge, die vom Süden weit in das ebene 


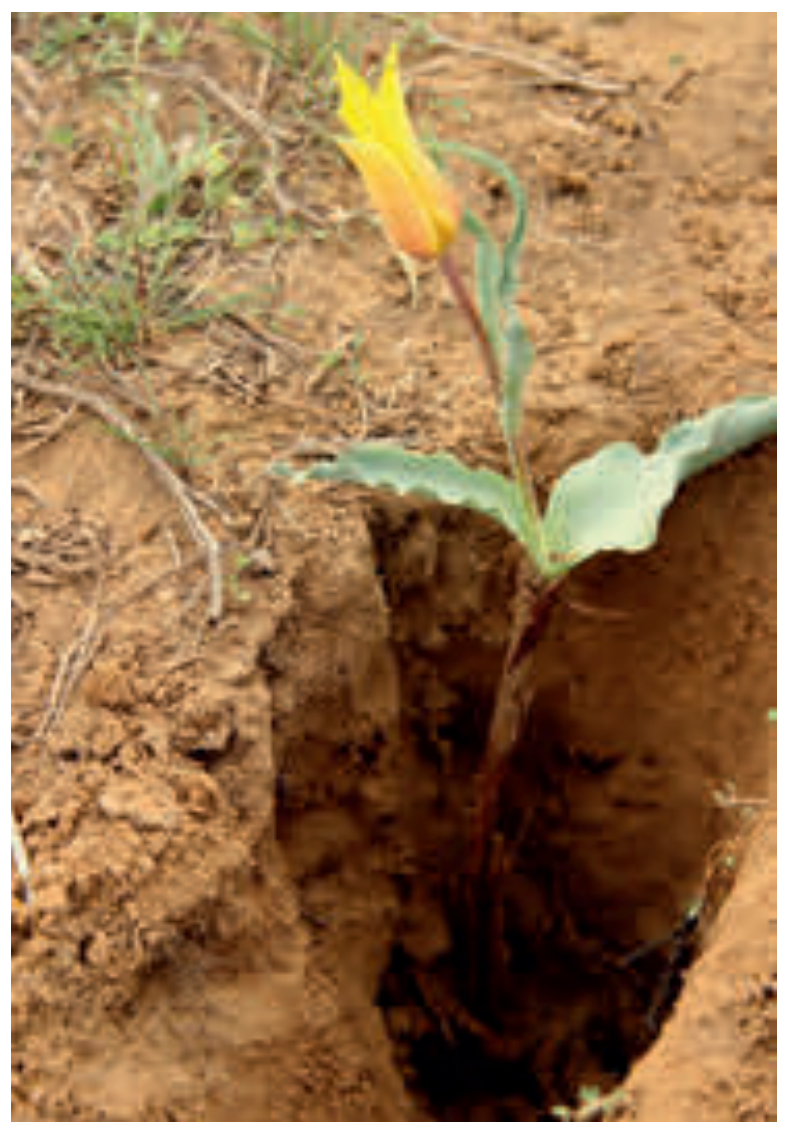

Wüstengebiet vordringen und die Wasserscheide zwischen dem Siebenstromland mit der Taukum- und der Mojynkum-Wüste bilden. In durchschnittlichen Frühjahrsmonaten fällt hier noch genug Regen, sodass die ausgedehnten Weideflächen grün erscheinen. An feuchten Rinnen fällt Lasiagrostis splendens, ein dem Pfriemengras (Stipa) nahestehendes Süßgras, auf, das bei der Auskleidung von Jurten früher von großer Bedeutung war. In dieser lebensfeindlichen Gegend nahe der Ortschaft Karabastau überraschen die bis zu 3000 Jahre alten Petroglyphen (Felsgravuren) von Tamgaly Tas. Dieses bedeutende Zeugnis prähistorischer Besiedelung auf den Chu-Ili-Bergen ist aber auch ein botanischer Pilgerort. Die wohl seltenste Tulpenart (Tulipa regelii) ist nur hier um Karabastau zu finden. Im zeitigen März erscheint ein Blattpaar mit auffallend welligen Blättern. Kurz danach zeigt sich eine etwa $15 \mathrm{~cm}$ hohe weiße Blüte mit gelbem Schlund. Benannt ist diese Tulpe nach dem deutschen Gärtner und Botaniker Eduard August von Regel, der 1875 bis zu seinem Tod 1897 Direktor des Bo-
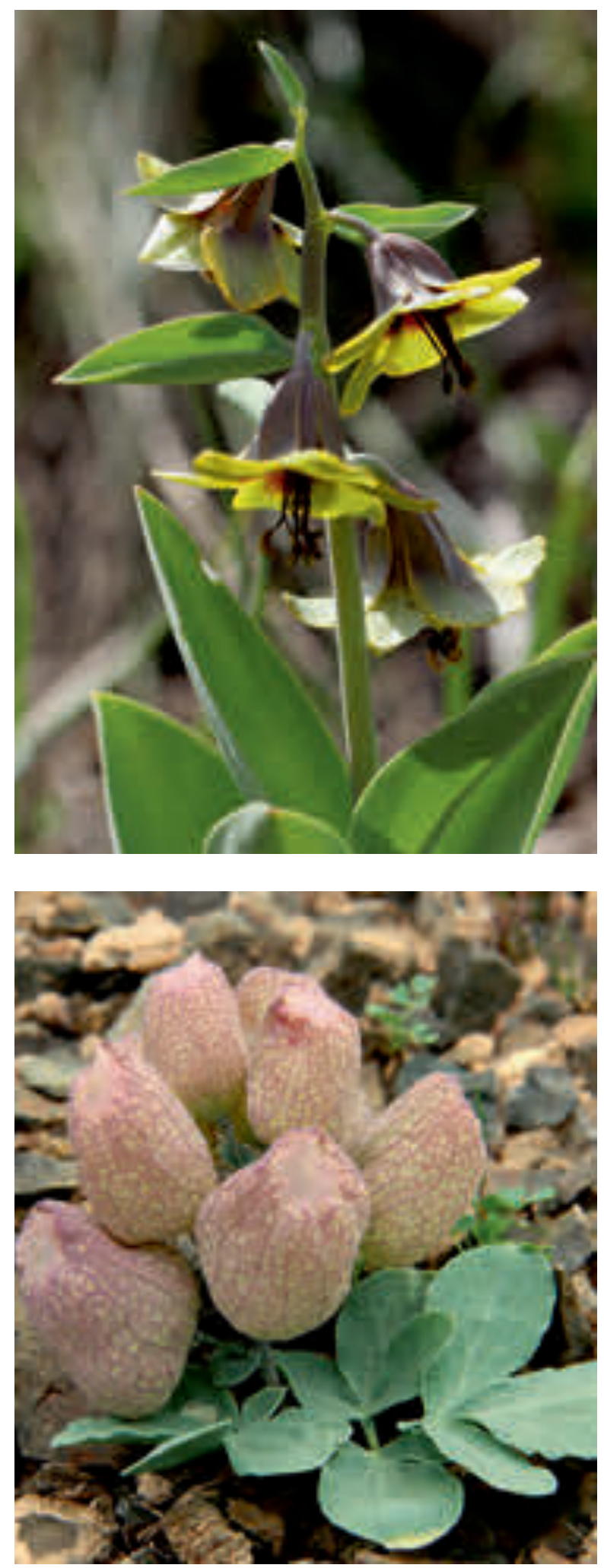

Abb. 4 (Seite 134): Talas Alatau mit Mt. Manas.

Abb. 5 (oben links): Tulipa buhseana.

Abb. 6 (oben rechts): Korolkowia sewerzowii.

Abb. 7 (unten): Leontice insertum. 


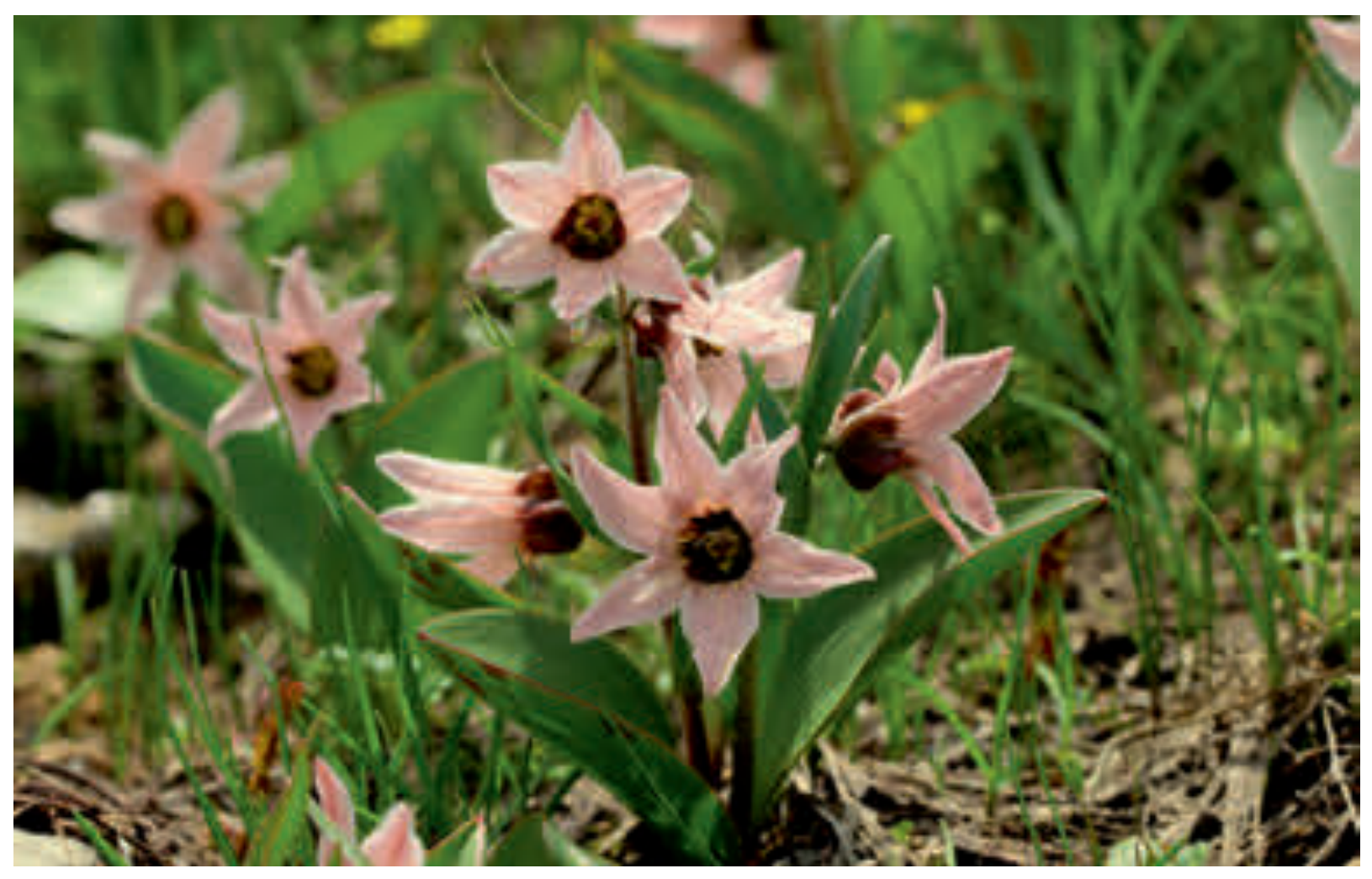

tanischen Gartens St. Petersburg war und zahlreiche, für das Zarenreich neu entdeckte Pflanzen, darunter viele Tulpenarten, beschrieb.

Die felsigen Bergrücken an den Ausläufern der Chu-Ili-Berge zeigen deutliche geologische Unterschiede, sodass auf kleinen Arealen ganz unterschiedliche Pflanzengesellschaften, unter anderem mit Lotwurz (Onosma gmelinii), Iris kuschakewiczii und blutroter Tulipa albertii, wachsen. Im April längst abgeblüht ist Leontice insertum, ein in Zentralasien vorkommendes krautiges Berberitzengewächs mit aufgeblasenen Früchten, die vom Wind vertragen werden. Weiter im Südwesten reichen die Karatau-Berge (Schwarze Berge), ausgehend vom Kirgischen Alatau, noch weiter in die angrenzenden Wüstengebiete. Auf den zerklüfteten, über $2000 \mathrm{~m}$ hohen Bergen sind im April noch kleine Schneeflecken zu sehen, und die Quellbäche führen viel Wasser. Doch kaum treten sie aus den Bergen, versickert das Wasser im Wüstenboden.

$\mathrm{Zu}$ den botanisch interessantesten Gebieten zählt das Berkara-Tal am Fuße des KaratauGebirges. Nur am Talgrund wachsen dichte Bestände von Fraxinus sogdianus und Acer sime- nowii. In Vollblüte stehen im April einzelne Bäume von Malus sieversii und Pyrus regelii, eine Wildbirne mit fünflappigen Blättern. Die Baum- und Strauchvegetation lockert an den Hängen rasch auf und Kleinsträucher wie $C e$ rasus tianschanica mit zartrosa Blüten und Dornbüsche von Hulthemia (Rosa) persica herrschen vor. Zwischen den feingliedrigen, zartgrünen Blättern von Prangos pabularis (Apiaceae) leuchten die roten, großen Blüten von Tulipa greigii. Der Weg verliert sich im groben Blockschutt, wo Allium karataviense, Steppenkerzen (Eremurus lactiflorus) und der dicht behaarte Astragalus atrovinosus blühen.

Kaum treten die Berge des Karatau zurück, breitet sich die scheinbar endlose MojynkumWüste aus. Die Fahrt zur Wüstenstadt Kumkent (Kum = Sand) führt vorbei an der einst blühenden Bergwerkstadt Shangatas, wo jetzt mit hohem technischen Aufwand und wenig Bergmännern Uran abgebaut wird und die großen Arbeitersiedlungen leer stehen. Vor dem Kysylköl (Roter See), einem Salzsee, wächst eine Tulpenart (Tulipa borszczowii), die sogar den salzigen Sandstürmen zu trotzen vermag. Die atemberaubende Landschaft um den Kysylköl 
kann nicht darüber hinwegtäuschen, dass nur wenige Pflanzen wie Caragana kirgisorum und Tamarix ramosissima hier überleben können. Für Vögel ist das Gebiet dagegen ideal, und im Frühsommer halten sich hier sehr viele Vögel auf.

\section{Nationalpark Aksu Zhabagly}

In der Ferne sind an klaren Tagen die im April noch schneebedeckten Berge der Hauptkette des Talas Alatau an der Grenze zu Kirgistan und Usbekistan zu erkennen. Das kleine Flüsschen Koksaj bildet mit einem bizarren Canyon die Grenze. Dahinter türmen sich mit dem Massiv des Manas (4484 m) die höchsten, bereits in Kirgistan befindlichen Berge auf. Bereits 1926 wurde der $850 \mathrm{~km}^{2}$ große Nationalpark Aksu Zhabagly eingerichtet. Der Talas Alatau, dieser westliche Ausläufer des Tianshan, zählt zu den artenreichsten Gebieten Zentralasiens.

Die Hochebene am Koksaj liegt auf ca. $1500 \mathrm{~m}$. Pflanzen, die in den Steppen und Wüstengebieten bereits im Frühling verblüht sind, entfalten hier im April gerade die volle Blütenpracht. Es gedeihen hier beispielsweise Iridodyctium kolpakowskiana, Iris ochroleuca und Corydalis ledebouriana. An den Felswänden des Talas-Flusses findet man die sehr seltene Steinbrechart Saxifraga alberti, die mit den Züchtungen desselben Namens nichts zu tun hat.

Die kleine Ortschaft Zhabagly ist wichtiger Ausgangspunkt für Wanderungen im Naturreservat. Etwas umständlich ist es, die Genehmigung am Eingang des Naturreservates zu erhalten. Zumindest unserer Exkursionsgruppe wurde leider ein völlig unwissender Ranger als Begleitung mitgegeben, der weder besondere Standorte noch andere brauchbare Hinweise geben konnte.

Abb. 8 (Seite 136): Rhinopetalum stenanthera.

Abb. 9 (oben): Allium atrosanguineum.

Abb. 10 (unten): Cistanche salsa.
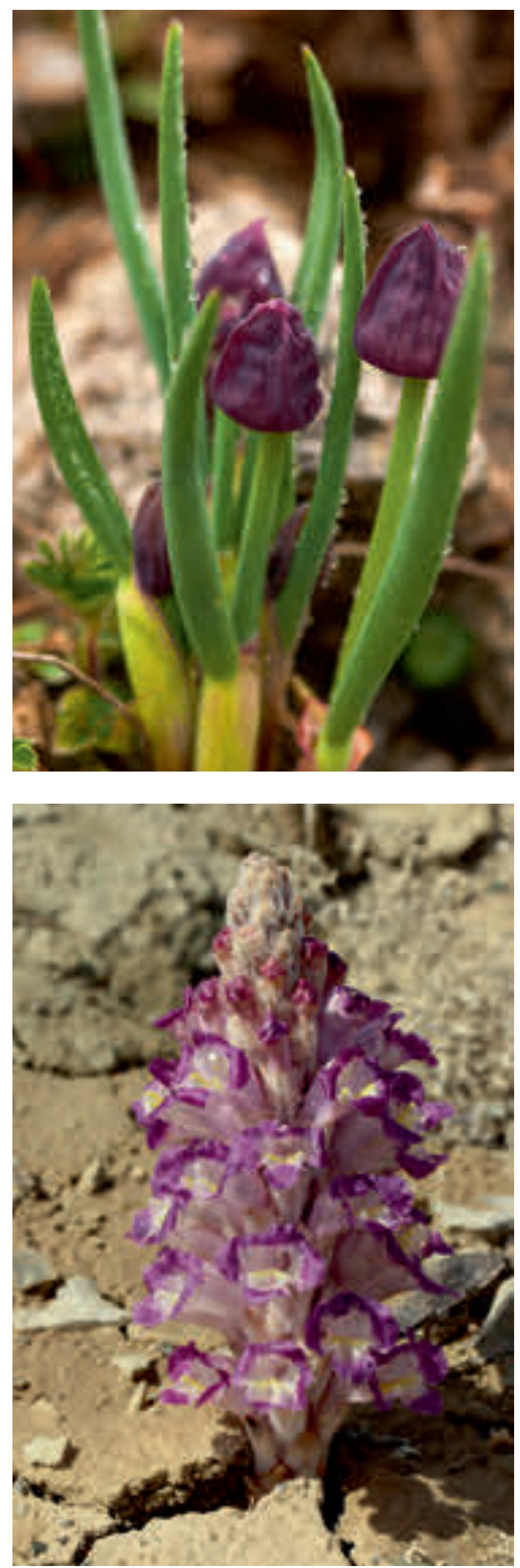

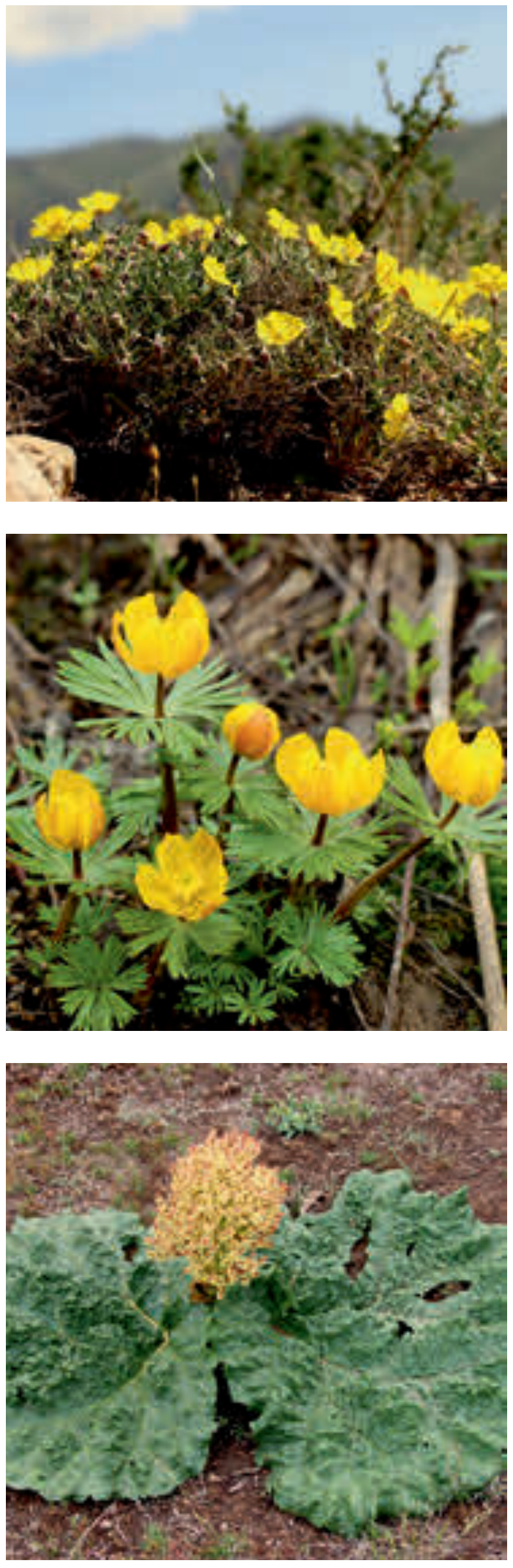

Der Weg zu einer etwa $1800 \mathrm{~m}$ hoch gelegenen Hütte erfüllt alle botanischen Träume. Schon nach wenigen Metern blühen am Wegrand Iris coerulea und Iris orchioides. Auf einem steilen Hang ist ein „Roter Teppich “ mit Tulipa greigii kaum zu übersehen. Fast immer als Einzelgehölz, fallen zwei Wacholderarten auf, die in der breitpyramidalen Wuchsform typisch für das ganze Gebiet Aksu Zhabagly sind: Juniperus semiglobosum mit vier oder mehr Samen pro Beerenzapfen und J. turcestanica mit zweisamigen Beerenzapfen. Heftiger Regen und Schneefall bis unter $2000 \mathrm{~m}$ herab haben auf unserer Exkursion manchen Pflanzen zugesetzt, trotzdem ist die Blütenvielfalt unglaublich.

Von gelblich-grün bis braunrot blühen die bis etwa $50 \mathrm{~cm}$ hohen und mit Fritillarien nahe verwandten Korolkowia sewerzowii. Korolkowia ist eine monotypische Gattung Zentralasiens. Die sternförmigen Blüten sind ein deutliches Unterscheidungsmerkmal zu Schachbrettblumen. In den Schneetälchen zeigen sich die ersten Blüten von Gymnospermium altaicum, wie die bereits erwähnte Knollenpflanze Leontice incerta, ebenfalls ein Vertreter der Berberidaceae. Zwischen den leuchtend gelben Blüten von Sibatheranthis longistipitata öffnen sich auch die ersten Blüten von Tulipa turcestanica und Crocus alatavicus. An den noch geschlossenen Blüten ist die für diese Art typische violette Zeichnung an der Außenseite der Petalen erkennbar.

Nicht zu Unrecht wird Tulipa kaufmanniana als "Seerosentulpe“ bezeichnet, die gegenüber der ebenfalls als Wildtulpe in unseren Gärten kultivierten Greigii-Tulpe höhere, doch etwas feuchtere Lagen bevorzugt. Die Fahrt zum berühmten Aksu-Canyon war der Gruppe wegen starker Regen- bzw. Schneefälle leider verwehrt, da die Straße nicht passierbar war. Doch die

Abb. 11 (oben): Helianthemum songoricum.

Abb. 13 (Mitte): Sibatheranthis longistipitata.

Abb. 14 (unten): Rheum tataricum.

Abb. 12 (Seite 139): Scharyn-Schlucht. 
über eine steile Felsenpiste führende Straße zum kleinen Dorf Rajevka auf die Hochebene mit einer unvergleichlich schönen Landschaft ist dann noch möglich. Sattgrün zeigen sich hier die Weidewiesen und die frisch bestellten Äcker. An den Ackerrändern blühen wie in einem Garten ausgepflanzt unzählige Scilla puschkinoides, Gagea bulbifera und Tulipa kaufmanniana. Die ebenen Flächen werden von canyonartigen Ausschwemmungen durchzogen, wodurch eine landwirtschaftliche Nutzung nicht möglich ist. In diesen Gräben blüht Rhinopetalum stenanthera.

\section{Nationalpark Ile-Alatau}

Um möglichst viel in einem derart großen Land kennenzulernen, bietet es sich an, die lange Fahrt mit der Turksib, einer Nebenlinie der Transsibirischen Eisenbahn, in der Nacht nach Almaty zurückzulegen. Vor den Toren Almatys kann ein zweiter Nationalpark kennengelernt werden. Der Ile-Alatau-Nationalpark beginnt bereits an der Stadtgrenze und reicht bis an die nahe Landesgrenze zu Kirgistan. Die nördlichen Teile der Stadt am Übergang zu den Step- pengebieten liegen auf ca. 500 m Höhe, während im Süden am Fuß des Transili-Alatau die Besiedlung bis etwa $1700 \mathrm{~m}$ reicht und auch an den Grenzen des Nationalparks nicht zu enden scheint. Gegenüber dem westlichen Talas-Alatau ist im zentralen Alatau das Klima etwas rauer und die winterliche Schneedecke reicht noch im April unter $2000 \mathrm{~m}$ herab. Der im Allgemeinen lang andauernde Winter mit ausreichend Schnee könnte zur Gefahr für dieses an besonderen Pflanzen so reiche Gebiet werden, wenn das Schigebiet wie geplant in die Schutzzonen des Nationalparks gebaut wird.

An den Hängen blüht hier im Frühling Malus sieversii. Der deutsche Botaniker CARL FrIEDRICH VON LEDEBOUR beschrieb 1833 in der Flora Altaica erstmals diesen Wildapfel als Pyrus sieversii. Durch neue Untersuchungen mittels Genanalysen konnte endgültig nachgewiesen werden, dass dieser Apfel als „Urapfel“ zu bezeichnen ist und somit Almaty zu Recht „Stadt des Apfels“ genannt werden kann. Auf den tiefgründigen, lehmigen Böden sind dichte Bestände von milchblauen Iris albertii und karmesinroten Blüten von Paeonia intermedia zu

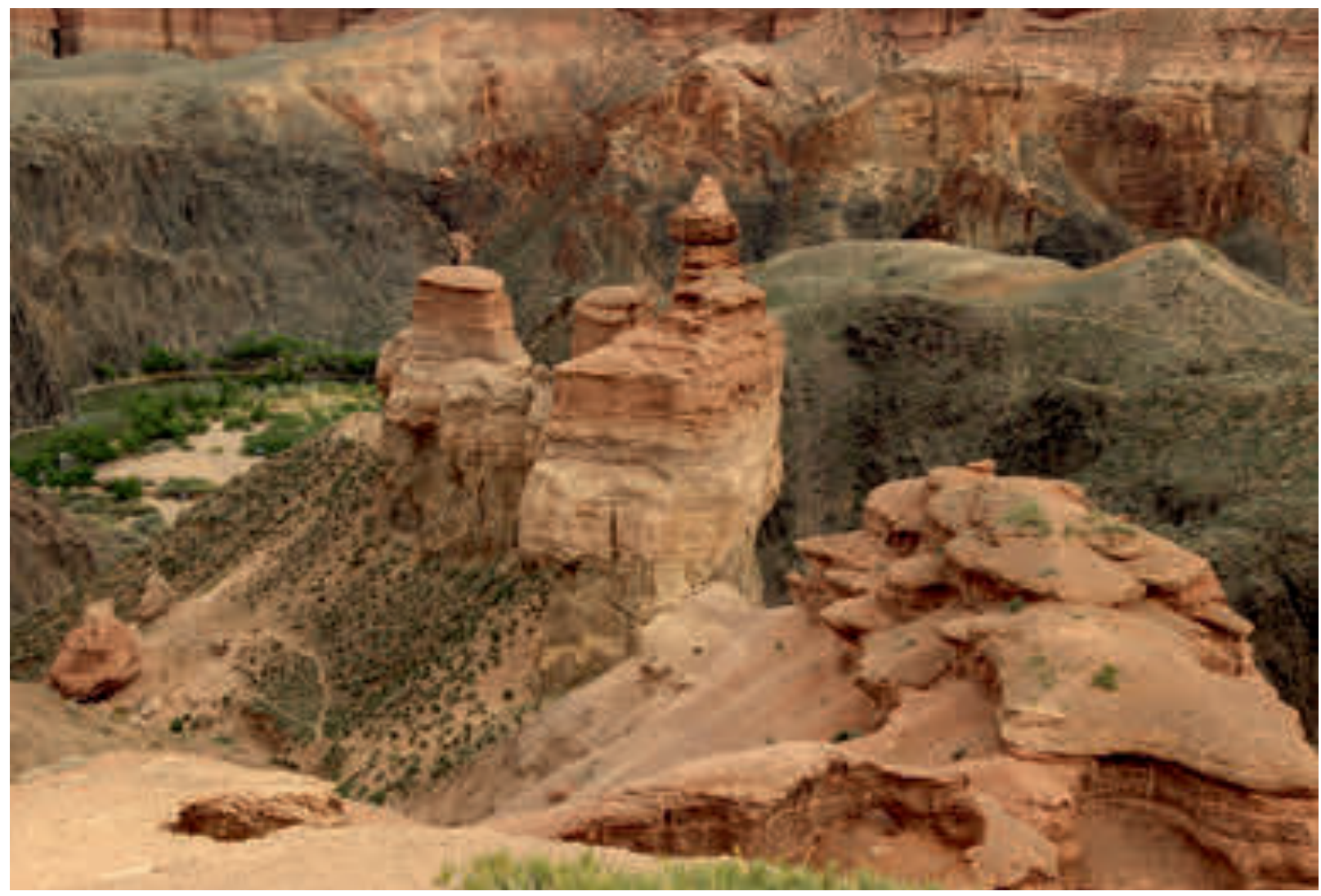


finden. Von dichten Tautropfen glitzern morgens die stark behaarten Blütenstände von Eremostachys molucelloides, ein etwa $30 \mathrm{~cm}$ hoher Lippenblütler mit strahlend gelben Blüten. An den Nordhängen des Transili-Alatau bilden die schmalkronigen Tianschan-Fichten (Picea schrenkiana) dichte Bestände, die gegen die Waldgrenze zu rasch auflockern und mit Tianshan-Birken (Betula tianschania) durchmischt sind.

Die Straße führt über den Großen Alamtiner-See $(2511 \mathrm{~m})$ zu einem ehemaligen Observatorium. Die Krummholzzone wird hier von Juniperus sibirica gebildet. $\mathrm{Zu}$ den ersten blühenden Frühlingsboten zählen Allium atrosanguineum, Pulsatilla campanella, Viola altaica und Crocus alatavicus. Nahe dem Großen Almatiner-See blühen nur etwa $20 \mathrm{~cm}$ hohe gelbe Tulpen mit braunen Streifen auf den Petalen. Diese Tulipa heterophylla bildet mit zwei weiteren Arten, die alle in den alpinen Regionen des Tian-Shan, Altai und der Mongolei vorkommen, die Sektion (Subgenus) Orithyia.

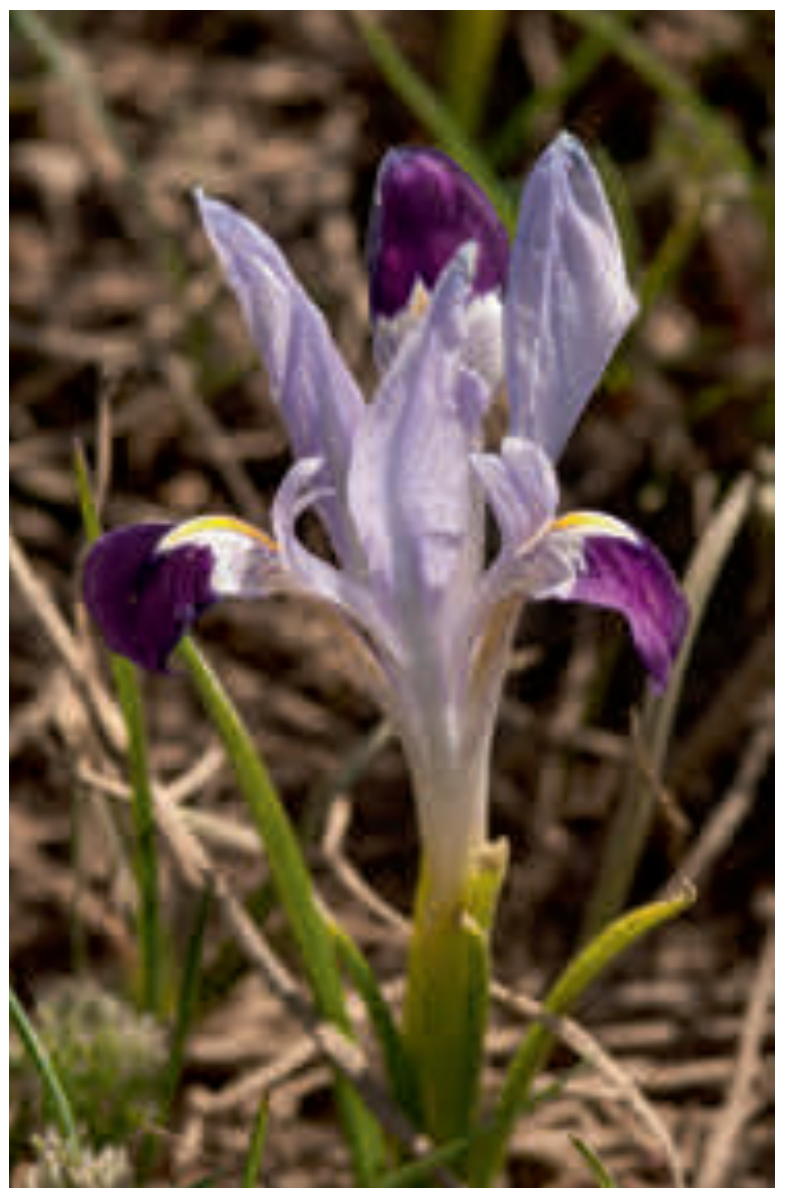

\section{Der einsame Südosten}

Die Straße von Almaty in den Osten führt zunächst durch das östliche Ili-Becken mit ausgedehntem Weideland. An feuchten Gräben sind die dichten Horste von Iris iliensis zu erkennen, die im April ihre blauen Blüten entfalten. Je weiter man aber nach Osten kommt, desto karger wird die Landschaft, bis schließlich Felswüsten überwiegen. Das große Dorf Bajseit, etwa $150 \mathrm{~km}$ östlich von Almaty, liegt am Übergang zu den Wüstengebieten. Die Hauptstraße ist gleichzeitig belebter Marktplatz mit der bunten Vielfalt typischer uigurischer Märkte, wo neben diversem Hausrat und Gemüse auch interessante Heilsteine angeboten werden.

An den kahlen Hügeln, die gleich nach dem Ort die Landschaft prägen, vermutet man kaum eine spannende Pflanzenwelt. Die Dornbuschvegetation besteht vor allem aus Bocksweizen (Atraphaxis spec.) mit rötlich geflügelten Nüsschen und lockeren Saxaul-Beständen. In früheren Erzählungen wird sehr oft vom „Baum der Wüste“ berichtet, der an den alten Handelsstraßen als wichtige Brennholzquelle zu finden war. Gnadenloser Raubbau hat diesen Bestand der "Saxaulwälder" so weit reduziert, dass Schutzprogramme und Aufforstungen zum Schutz gegen die Erosion eingeleitet werden mussten. Dennoch wird Saxaul weiterhin für die an den Rastplätzen und Märkten allgegenwärtigen Schaschlikspieße abgeholzt. Es ist ein sehr empfindliches Ökosystem mit wenigen sehr gut angepassten Begleitpflanzen wie Nitraria-Sträuchern und auffallenden Schmarotzerpflanzen wie die den Orobanchen nahestehende Cistanche salsa oder der Malteserschwamm (Cynomorium songoricum). Zwischen den Felsblöcken leuchten die gelben Blüten einer dem ariden Klima bestens angepasste Sonnenröschen-Art (Helianthemum songoricum), die in den Zentralasiatischen Steppen und Halbwüsten verbreitet ist. Hier kommt auch Wüsten-

Abb. 15: Iridodyctium kolpakowaskianum.

Abb. 16 (Seite 141 oben): Cynomorium songaricum.

Abb. 17 (Seite 141 unten): Tulipa dasystemon. 
Helmkraut (Scutellaria transiliensis) mit gelber Lippe und rotem Helm vor.

Zwischen Steinblöcken ducken sich die sukkulenten Rosetten von Orostachys thyrsiflora. Die Hügelketten schließen immer enger zu einer wasserlosen Enge zusammen und bilden die Kokpekschlucht. Unzählige 50 bis $60 \mathrm{~cm}$ hohe hellorange Blütenstände von Eremurus fuscus stehen wie bunte Kerzen an den steilen Hängen. An der Straße in der Schlucht bieten Händler in großen Büscheln Allium galanthum an, eine als Gemüse und Heilpflanze sehr geschätzte Lauchart, die offenbar in der Umgebung in großen Mengen ausgegraben wird. Nach der Kokpek-Schlucht gelangt man auf die Sogety-Hochebene. Ein pittoreskes Farbenspiel bietet dem Betrachter die Weite der Wüste, wenn Syrenia, ein kleiner Kreuzblütler, riesige gelbe Teppiche aus unzähligen, kaum $30 \mathrm{~cm}$ hohen Pflänzchen bildet. Die Eintönigkeit durchbricht auch der Tatarische Rhabarber (Rheum tataricum) mit seinen roten Blütenständen, dessen etwa $1 \mathrm{~m}^{2}$ großen Blätter dem Sand aufliegen. Entlang der Sandpiste treten häufig kleine Bestände der an das Wüstenklima bestens angepassten Iris scariosa auf.

Die Ebene bricht plötzlich in eine Schlucht $a b$, die der Scharyn-Fluss in das weiche rotbraune Gestein gegraben hat. Der Scharyn-Canyon mit seinen vielen Sandsteintürmchen und erodierten Felsformationen ist eines der beeindruckendsten Naturwunder Kasachstans und wird oft mit dem Grand Canyon der USA verglichen.

Gegen Südosten in das kirgisisch-chinesische Grenzgebiet treten die Vorberge des Alatau immer weiter heran. Bis zum Kegenpass (2300 m) im Ketmen-Gebirge kann man ohne gesonderte Genehmigung fahren. Auf den Felskuppen finden sich bereits einige Elemente des östlichen Tian-Shan wie z. B. Androsace sericea, Iris tianschanica ssp. loczyi und Tulipa iliensis. An den windgefegten Kanten schmiegen sich Juniperus turkestanica und Lonicera humilis an die Felsen.

Kasachstan ist noch wenig touristisch erschlossen. Verzichtet man auf die Bequemlichkeiten des Massentourismus, kann man in die-
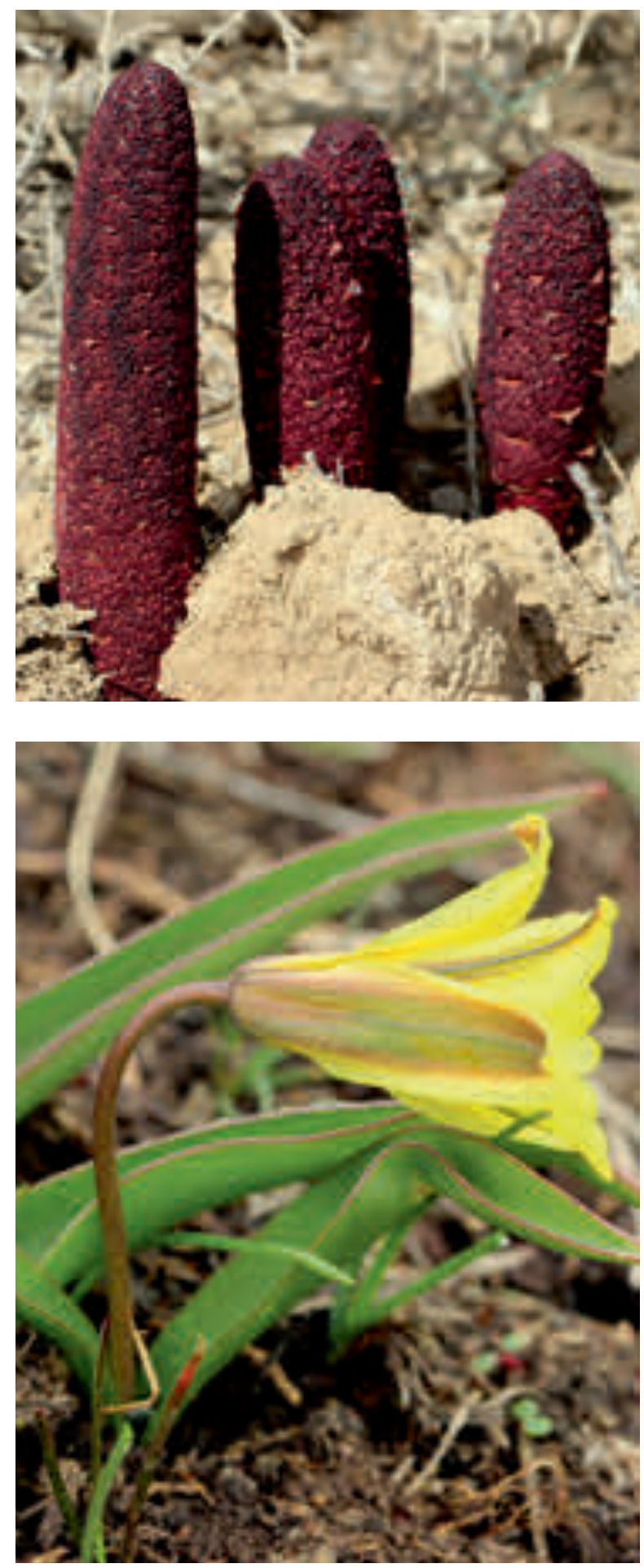

sem zentralasiatischen Land eine unglaubliche Vielfalt an unberührter Natur und eine unverfälschte Gastfreundschaft erleben. Als Reiseziel für Interessierte an der Tier- und Pflanzenwelt bleibt Kasachstan vorerst ein Geheimtyp.

Literatur

Everett, D. 2013: The Genus Tulipa. - Kew. 